TRANSACTIONS OF THE

AMERICAN MATHEMATICAL SOCIETY

Volume 363, Number 4, April 2011, Pages 2029-2046

S 0002-9947(2010)04980-3

Article electronically published on October 25, 2010

\title{
FRAMES AND DEGENERATIONS OF MONOMIAL RESOLUTIONS
}

\author{
IRENA PEEVA AND MAURICIO VELASCO
}

AbStRACt. We study free resolutions of monomial ideals in a polynomial ring.

\section{INTRODUCTION}

We study the structure of (minimal) free resolutions of monomial ideals over a polynomial ring. This has been a very active area of research, and a number of new ideas and approaches were introduced in the last decade.

Homogenization and dehomogenization of ideals are widely used (for example, to relate the defining ideals of affine and projective varieties). We introduce homogenization of complexes of vector spaces. Given a monomial ideal $M$, our $M$ homogenization Construction 3.2 describes an algorithm which transforms a complex of vector spaces into a complex of multigraded free modules. The main point is that we determine the multidegrees of the basis vectors in the complex in the optimal smallest way. An important virtue of the construction is that it guarantees that the multidegrees of the basis elements are in the lcm-lattice of $M$.

A frame of a monomial free resolution is a complex of vector spaces obtained by dehomogenizing the resolution with respect to a fixed basis. Theorem 4.14 shows that if the resolution is minimal, then any of its frames encodes the structure of the resolution entirely (in the sense that homogenizing the frame produces the resolution). The key idea in our paper is that the problem of constructing a minimal monomial free resolution is equivalent to the problem of building any of its frames. This reduces the core problem of classification of minimal free resolutions of monomial ideals to the problem of classification of their frames. It also opens up the following approach: in order to obtain the minimal free resolution of a monomial ideal it suffices to build any of its frames.

A strong advantage of this approach is that every minimal free resolution can be obtained using our construction, while it is known by [14 that cellular resolutions do not give all minimal free resolutions.

The approach seems to have the potential to extend the class of combinatorial and topological objects which can be used for constructions of monomial resolutions. At this point, there are three main known sources of frames: homology and

Received by the editors August 8, 2008 and, in revised form, June 14, 2009.

2000 Mathematics Subject Classification. Primary 13F20.

Key words and phrases. Resolutions of monomial ideals. 
cohomology chain complexes from algebraic topology (which yield cellular and cocellular resolutions), dehomogenization of resolutions (see Section 4), and in some cases it is possible to construct frames directly (see Theorems 6.1 and 7.1).

Special cases of this construction have been used by several authors: for example, this is how simplicial resolutions [2], cellular resolutions [3, the Buchsbaum-Rim resolutions [5, and the cocellular resolutions [11] are built; it is also used in [4, 13. A strong advantage of our approach is that it does not need a special choice of basis in the resolution (that is, any frame can be used), while all the special cases listed above need it. This is important because the potential for applications would have decreased dramatically if one needed to choose a good basis first. Here is an example of how the choice of basis caused difficulties in the setting of cellular resolutions. Consider the minimal free resolution $\mathbf{X}$ of a power of the maximal ideal. A well-known resolution with many applications is the Eliahou-Kervaire resolution [6], which gives an explicit formula for the differential in a special basis of $\mathbf{X}$. It is natural to ask if this resolution is cellular. Batzies and Welker [1] proved that there exists a choice of basis in $\mathbf{X}$ that supports a cellular structure. Later, Sinefakopoluos and Corso-Nagel obtained two other choices of basis in $\mathbf{X}$ that support a cellular structure. However, none of these cellular structures used the basis that the well-known Eliahou-Kervaire resolution uses. Finally, Mermin 10. was able to show that the basis in the Eliahou-Kervaire resolution can be used for a cellular structure. It should be noted that changing the basis of a cellular resolution completely destroys the cellular structure.

We introduce the concept of $f$-homogenization of a monomial free resolution, and we compare it to the construction of $f$-degeneration from $[8$. While $f$-homogenization and $f$-degeneration may at first sight look quite similar, Example 4.11 shows that they can differ if applied to a nonminimal resolution. Example 7.3 brings up another important point: the choice of basis. Corollary 4.15 shows that a good choice of basis exists in some cases.

Our constructions and results in Sections 3 and 4 provide a framework which allows us to treat several important constructions and results on monomial resolutions in [2, 3], 8] as particular cases. This is explained in more detail in Remarks 3.3, $3.9,3.11,4.2,4.7,4.9$.

The results in Sections 5, 6, and 7 are applications of the techniques developed in Sections 3 and 4. On the one hand, the applications are interesting on their own, and on the other hand, they provide an illustration of our methods.

In Section 5, we study Scarf complexes. Scarf simplicial complexes and resolutions were introduced in 2. It is natural to ask what simplicial complexes appear as Scarf complexes of monomial ideals, and what simplicial complexes appear as Scarf complexes of Scarf ideals. In Theorem 5.3(1), we show that every finite simplicial complex, except the boundary of a simplex, is the Scarf complex of some monomial ideal. It was observed in [2, Lemma 2.1] that if a simplicial complex $\Delta$ is the Scarf complex of a Scarf ideal, then $\Delta$ is acyclic; furthermore, Example 5.2 in 2 shows that $\Delta$ need not be pure or shellable. In Theorem 5.3(2), we show that every finite acyclic simplicial complex is the Scarf complex of a Scarf ideal. As a consequence, we characterize the sequences of Betti numbers of Scarf ideals in Corollary 5.4.

In the proof of Theorem 5.3 we introduce nearly Scarf ideals. This is a class of monomial ideals with highly structured minimal free resolutions. The lcm-lattice of such a monomial ideal consists of the multidegrees of the faces of its Scarf complex 
and a top element (which is the lcm of all the minimal monomial generators of the ideal). In Theorem 6.1, we construct the minimal free resolution of any monomial ideal with such an lcm-lattice. In Corollary 6.3 we list the numerical invariants of the minimal free resolution of a nearly Scarf ideal.

In Section 7, we obtain a lower bound on the Betti numbers of a monomial ideal in terms of its Scarf complex. The bound is sharp: it is attained by every nearly Scarf ideal. Furthermore, in Theorem 7.1(2), we describe the structure of the minimal free resolution of every monomial ideal with minimal Betti numbers among all monomial ideals with a fixed Scarf complex.

There are very few classes of monomial ideals (for example, Borel ideals [6] and Scarf ideals [2]) for which the minimal free resolution is known explicitly. Theorems 6.1 and 7.1 provide two new such classes: nearly Scarf ideals and ideals with minimal Betti numbers.

Next we discuss Theorem 6.1, which is our main application. Resolutions supported by a simplicial complex were introduced in [2]. This was generalized to cellular resolutions, introduced in [3]; such a resolution is supported by a regular cell complex. Furthermore, the class was generalized in $[9]$ to include all resolutions supported by CW-complexes. The theory of cellular resolutions has been a very active area of research. As more and more cellular resolutions are found, the following fundamental question had been open:

Question 1.1. Is it true that all minimal free resolutions of monomial ideals are $C W$-cellular?

It was unclear whether to expect a positive or a negative answer. Question 1.1 had been very challenging, and the challenge was that no one had any clue where to look for counterexamples. Our main application is the introduction of nearly Scarf ideals and the description of their minimal free resolutions in Theorem 6.1. Velasco shows in 14 that this class of ideals contains the following two types of examples:

(1) There exist nearly Scarf ideals whose minimal free resolutions are not CWcellular.

(2) There exist nearly Scarf ideals whose minimal free resolutions are CWcellular but are not supported by any regular cellular complex.

This solved Question 1.1. The resolutions in our Theorem 6.1 provide the only known examples of type (1) and many natural examples of type (2). The theory we develop in Sections 3 and 4 is essentially very simple; nevertheless, it has the power to lead to the resolutions of nearly Scarf ideals, which are fundamentally new.

\section{Preliminaries}

We introduce notation which will be used throughout the paper. Let $k$ be a field and let $M$ stand for a monomial ideal in the polynomial ring $S=k\left[x_{1}, \ldots, x_{n}\right]$ minimally generated by monomials $m_{1}, \ldots, m_{r}$.

2.1. Grading. The polynomial ring $S$ is $\mathbf{N}^{n}$-graded by setting $\operatorname{deg}\left(x_{i}\right)$ to be the $i$ 'th standard vector in $\mathbf{N}^{n}$. Often we say that $S$ is multigraded instead of $\mathbf{N}^{n_{-}}$ graded, and we say multidegree instead of $\mathbf{N}^{n}$-degree. $\overline{\text { For every } \mathbf{a}}=\left(a_{1}, \ldots, a_{n}\right) \in$ $\mathbf{N}^{n}$ there exists a unique monomial of degree $\mathbf{a}$, namely $\mathbf{x}^{\mathbf{a}}=x_{1}^{a_{1}} \cdots x_{n}^{a_{n}}$. If an element $g$ (say in a module) has $\mathbf{N}^{n}$-degree $\mathbf{a}$, then we say that it has $\underline{\text { multidegree }}$ 
$\mathbf{x}^{\mathbf{a}}$ and denote $\operatorname{deg}(g)=\mathbf{x}^{\mathbf{a}}$. Denote by $S\left(-\mathbf{x}^{\mathbf{a}}\right)$ the free $S$-module generated by one element in multidegree $\mathbf{x}^{\mathbf{a}}$.

Every monomial ideal is multihomogeneous. Hence, there exists a minimal free resolution of $S / M$ over $S$ which is multigraded. Thus, we have multigraded $\underline{\text { Betti }}$ numbers

$$
b_{i, m}(S / M)=\operatorname{dim}_{k} \operatorname{Tor}_{i, m}^{S}(S / M, k) \text { for } i \geq 0, m \text { a monomial. }
$$

Therefore, the resolution can be written as

$$
0 \longrightarrow \ldots \longrightarrow \bigoplus_{m} S^{b_{3, m}}(-m) \longrightarrow \bigoplus_{m} S^{b_{2, m}}(-m) \longrightarrow \bigoplus_{m} S^{b_{1, m}}(-m) \longrightarrow S,
$$

where the sum runs over all monomials $m$.

We denote by $L_{M}$ the lattice with elements labeled by the least common multiples of subsets of $m_{1}, \ldots, m_{r}$ ordered by divisibility. The atoms in $L_{M}$ are $m_{1}, \ldots, m_{r}$; the top element is $\operatorname{lcm}\left(m_{1}, \ldots, m_{r}\right)$. The bottom element is 1 regarded as the $\mathrm{lcm}$ of the empty set. The least common multiple of elements in $L_{M}$ is their join. Following [8] we call $L_{M}$ the $\underline{\text { lcm-lattice }}$ of $M$. For $m \in L_{M}$ we denote by $(1, m)$ the open lower interval in $L_{M}$ below $m$; it consists of all nonunit monomials in $L_{M}$ that strictly divide $m$. The following result is proved in 8 :

Theorem 2.2. For $i \geq 1$ we have

$$
b_{i, m}(S / M)= \begin{cases}\operatorname{dim} \tilde{\mathrm{H}}_{i-2}((1, m) ; k) & \text { if } m \in L_{M}, \\ 0 & \text { if } m \notin L_{M} .\end{cases}
$$

\section{Homogenization AND DEHOMOGENiZATION OF COMPLEXES}

As stated in [11, beginning of Section 3], the paper [11 provides "a handy new matrix notation for maps": namely, the notions of a monomial matrix [11, 3.2] and coefficient matrix provide a mathematical language that one can use while working with multigraded resolutions. In the next construction, we introduce our own terminology, which we find more suitable for our purposes.

Construction 3.1. Let $\mathbf{U}$ be a complex of finite $k$-vector spaces with differential $\partial$ and a fixed basis, such that

$\circ U_{i}=0$ for $i<0$ and $i \gg 0$,

$\circ U_{0}=k$,

$\circ U_{1}=k^{r}$

$\circ \partial\left(w_{j}\right)=1$ for each basis vector $w_{j}$ in $U_{1}=k^{r}$.

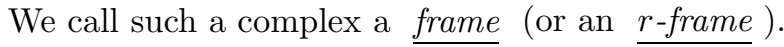

Let $\mathbf{G}$ be a multigraded complex of finitely generated free multigraded modules with differential $d$ and a fixed multihomogeneous basis with multidegrees in $L_{M}$, such that

$\circ G_{i}=0$ for $i<0$ and $i \gg 0$,

$\circ G_{0}=S$,

$\circ G_{1}=S\left(-m_{1}\right) \oplus \cdots \oplus S\left(-m_{r}\right)$,

$\circ d\left(w_{j}\right)=m_{j}$ for each basis element $w_{j}$ of $G_{1}$.

We call such a complex an $\underline{M \text {-complex }}$. 
The main construction in this section is:

Construction 3.2. Let $\mathbf{U}$ be an $r$-frame. We will construct by induction on the homological degree an $M$-complex $\mathbf{G}$ of free $S$-modules with differential $d$ and call it the $M$-homogenization of $\mathbf{U}$.

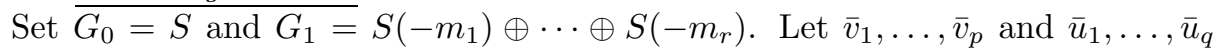
be the given bases of $U_{i}$ and $U_{i-1}$, respectively. Let $u_{1}, \ldots, u_{q}$ be the basis of $G_{i-1}=S^{q}$ chosen on the previous step of the induction. Introduce $v_{1}, \ldots, v_{p}$ that will be a basis of $G_{i}=S^{p}$. If $\partial\left(\bar{v}_{j}\right)=\sum_{1 \leq s \leq q} \alpha_{s j} \bar{u}_{s}$ with $\alpha_{s j} \in k$, then set

$$
\begin{aligned}
\operatorname{deg}\left(v_{j}\right) & =\operatorname{lcm}\left(\operatorname{deg}\left(u_{s}\right) \mid \alpha_{s j} \neq 0\right), \text { note that } \operatorname{lcm}(\emptyset)=1, \\
G_{i} & =\bigoplus_{1 \leq j \leq p} S\left(-\operatorname{deg}\left(v_{j}\right)\right), \\
d\left(v_{j}\right) & =\sum_{1 \leq s \leq q} \alpha_{s j} \frac{\operatorname{deg}\left(v_{j}\right)}{\operatorname{deg}\left(u_{s}\right)} u_{s} .
\end{aligned}
$$

We have that Coker $\left(d_{1}\right)=S / M$. The differential $d$ is multihomogeneous by construction. Straightforward verification shows that $\mathbf{G}$ is a complex. We say that the complex $\mathbf{G}$ is obtained from $\mathbf{U}$ by $M$-homogenization .

Remark 3.3. Simplicial resolutions, introduced in [2], are obtained by $M$ homogenizing the augmented oriented chain complex of a simplicial complex. Cellular resolutions, introduced in 3], are obtained by $M$-homogenizing the augmented oriented chain complex of a regular cell complex. CW-cellular resolutions, introduced in [9], are obtained by $M$-homogenizing the complex computing the homology of a CW-complex. In [5, Section 4], the Buchsbaum-Rim complex is homogenized in order to obtain a free resolution of a multigraded finitely generated module. Cocellular resolutions in [11] are obtained by $M$-homogenizing a cochain complex. The construction is also used in 4], [13].

Example 3.4. Consider the monomial ideal $L=\left(x^{5}, x y, y^{5}\right)$ in the polynomial ring $A=k[x, y]$ and the 3 -frame

$$
0 \longrightarrow k \stackrel{\left(\begin{array}{l}
1 \\
1 \\
1
\end{array}\right)}{\longrightarrow} k^{2} \stackrel{\left(\begin{array}{ccc}
-1 & 0 & 1 \\
1 & -1 & 0 \\
0 & 1 & -1
\end{array}\right)}{\longrightarrow} k^{3} \stackrel{\left(\begin{array}{l}
1 \\
1 \\
1
\end{array}\right)}{\longrightarrow} k .
$$

The $L$-homogenization of this frame is

$$
0 \rightarrow A\left(-x^{5} y^{5}\right) \stackrel{\left(\begin{array}{c}
y^{4} \\
x^{4} \\
1
\end{array}\right)}{\longrightarrow} A\left(-x^{5} y\right) \oplus A\left(-x y^{5}\right) \oplus A\left(-x^{5} y^{5}\right) \frac{\left(\begin{array}{ccc}
-y & 0 & y^{5} \\
x^{4} & -y^{4} & 0 \\
0 & x & -x^{5}
\end{array}\right)}{\longrightarrow}
$$

Construction 3.5. Let $\mathbf{G}$ be an $M$-complex. We call

$$
\mathbf{U}=\mathbf{G} \otimes S /\left(x_{1}-1, \ldots, x_{n}-1\right)
$$

the frame of $\mathbf{G}$ (or the dehomogenization of $\mathbf{G}$ ). Thus, $\mathbf{U}$ is a finite complex of finite $k$-vector spaces with fixed basis and its differential matrices are obtained 
by setting $x_{1}=1, \ldots, x_{n}=1$ in the differential matrices of $\mathbf{G}$. We say that the complex $\mathbf{U}$ is obtained from $\mathbf{G}$ by dehomogenization. Observe that:

Proposition 3.6. If $\mathbf{G}$ is the $M$-homogenization of a frame $\mathbf{U}$, then $\mathbf{U}$ is the frame of $\mathbf{G}$.

Construction 3.7. Let $\mathbf{G}$ be an $M$-complex, and let $m \in M$ be a monomial. Denote by $\mathbf{G}(\leq m)$ the subcomplex of $\mathbf{G}$ that is generated by the multihomogeneous basis elements of multidegrees dividing $m$. Set $v=\operatorname{lcm}\left(m_{i} \mid m_{i}\right.$ divides $\left.m\right)$. Then $\mathbf{G}(\leq m)=\mathbf{G}(\leq v)$ because all the basis elements of $\mathbf{G}$ have multidegrees in $L_{M}$ by Construction 3.1 .

The following simple criterion for exactness is very useful.

Theorem 3.8. Let $\mathbf{G}$ be an $M$-complex.

(1) For each monomial $m \in M$, the component of $\mathbf{G}$ of multidegree $m$ is isomorphic to the frame of the complex $\mathbf{G}(\leq m)$.

(2) The complex $\mathbf{G}$ is a free multigraded resolution of $S / M$ if and only if for all multidegrees $m \in L_{M}$ the frame of the complex $\mathbf{G}(\leq m)$ is exact.

Proof. Let $t$ be an element in the fixed multihomogeneous basis of $\mathbf{G}$. Denote by $P$ the free multigraded module generated by $t$. We have that $P$ contributes to the component of $\mathbf{G}$ of multidegree $m$ if and only if the multidegree $\operatorname{deg}(t)$ divides $m$; in this case, $P$ contributes the one-dimensional vector space spanned by the element $\frac{m}{\operatorname{deg}(t)} t$. Therefore, the component of $\mathbf{G}$ of multidegree $m$ is isomorphic to the frame of the complex $\mathbf{G}(\leq m)$. We have proved (1).

We will prove (2). The complex $\mathbf{G}$ is multigraded, so it suffices to check exactness in each multidegree. Note that $G_{0} / d\left(G_{1}\right)=S / M$. Therefore, it suffices to check exactness in each multidegree $m \in M$. By (1), it follows that the complex $\mathbf{G}$ is exact if and only if for all multidegrees $m \in M$ the frame of the complex $\mathbf{G}(\leq m)$ is exact.

Now, let $m \in M$ be a multidegree. Set $v=\operatorname{lcm}\left(m_{i} \mid m_{i}\right.$ divides $\left.m\right)$. Then $\mathbf{G}(\leq m)=\mathbf{G}(\leq v)$ because all the basis elements of $\mathbf{G}$ have multidegrees in $L_{M}$ by Construction 3.1. Therefore, it suffices to consider only the multidegrees in $L_{M}$.

Remark 3.9. Special versions (tailored to the specific situation in each case) of the criterion in Theorem 3.8 and its proof have been used several times before, for example in [2, proof of Lemma 2.2], 3, proof of Proposition 1.2], 5, proof of Theorem 4.5]. We present a general version. Note that in our version one has to check only finitely many multidegrees (namely the multidegrees in the lcm-lattice).

The next result shows that a free resolution $\mathbf{F}_{M}$ of $S / M$ contains as subcomplexes the minimal free resolutions for certain smaller monomial ideals.

Proposition 3.10. Let $u \in M$ be a monomial. Consider the monomial ideal $\left(M_{\leq u}\right)$ generated by the monomials $\left\{m_{i} \mid m_{i}\right.$ divides $\left.u\right\}$. Fix a multihomogeneous basis of a multigraded free resolution $\mathbf{F}_{M}$ of $S / M$.

(1) The subcomplex $\mathbf{F}_{M}(\leq u)$ is a free multigraded resolution of $S /\left(M_{\leq u}\right)$. If $\mathbf{F}_{M}$ is minimal, then so is $\mathbf{F}_{M}(\leq u)$.

(2) If $\mathbf{F}_{M}$ is the minimal free multigraded resolution of $S / M$, then the subcomplex $\mathbf{F}_{M}(\leq u)$ is independent of the choice of basis. 
Proof. (1) First, note that replacing $u$ by $\operatorname{lcm}\left(m_{i} \mid m_{i}\right.$ divides $u$ ) changes neither the ideal $\left(M_{\leq u}\right)$ nor the complex $\mathbf{F}_{M}(\leq u)$. So, we can assume that $u \in L_{M}$. By Theorem 3.8, it suffices to show that for every $m \in L_{\left(M_{\leq u}\right)}$, the frame of the complex $\left(\mathbf{F}_{M}(\leq u)\right)(\leq m)$ is exact. The frame of $\left(\mathbf{F}_{M}(\leq u)\right)(\leq m)$ is equal to the frame of $\mathbf{F}_{M}(\leq u \wedge m)$, where $u \wedge m$ is the meet of $u$ and $m$ in the lcm-lattice $L_{M}$. Since $\mathbf{F}_{M}$ is exact, by Theorem 3.8 it follows that the frame of $\mathbf{F}_{M}(\leq u \wedge m)$ is exact.

(2) holds because the multidegrees of the basis elements in $\mathbf{F}_{M}$ are the same in any choice of basis; they are determined by the multigraded Betti numbers.

Remark 3.11. Proposition 3.10 was proved independently in [7] and [11. The proof presented above is essentially the same as the proof of [11, Proposition 3.27(2)], and we include it for completeness.

Example 3.12. We illustrate Proposition [3.10. Let $A=k[x, y, z], T=\left(x^{2}, x y, x z\right.$, $\left.y^{2}\right)$, and $m=x y z$. Then $\left(T_{\leq x y z}\right)=(x y, x z)$. The minimal free multigraded resolution of $A / T$ is

$\mathbf{F}_{T}: \quad 0 \rightarrow A \stackrel{\left(\begin{array}{c}z \\ x \\ -y \\ 0\end{array}\right)}{\longrightarrow} A^{4} \stackrel{\left(\begin{array}{cccc}y & 0 & z & 0 \\ -x & z & 0 & y \\ 0 & -y & -x & 0 \\ 0 & 0 & 0 & -x\end{array}\right)}{\longrightarrow} A^{4} \stackrel{\left(\begin{array}{llll}x^{2} & x y & x z & y^{2}\end{array}\right)}{\longrightarrow} A \rightarrow 0$.

The subcomplex $\mathbf{F}_{T}(\leq x y z)$ is

$$
\left(\mathbf{F}_{T}\right)(\leq x y z): \quad 0 \rightarrow A \stackrel{\left(\begin{array}{c}
z \\
-y
\end{array}\right)}{\longrightarrow} A^{2} \stackrel{(x y \quad x z)}{\longrightarrow} A \rightarrow 0 .
$$

It is the minimal free multigraded resolution of $A /(x y, x z)$.

\section{Degeneration}

Definition 4.1. Let $M^{\prime}$ be a monomial ideal in a polynomial ring $S^{\prime}$ over the same ground field $k$. We say that $M$ is a reduction of $M^{\prime}$ if there exists a map $f: L_{M^{\prime}} \rightarrow L_{M}$ which is a bijection on the atoms and preserves lcm's. In what follows, we will use $f$ to order the minimal monomial generators $m_{1}^{\prime}, \ldots, m_{r}^{\prime}$ of $M^{\prime}$ so that $f\left(m_{i}^{\prime}\right)=m_{i}$ for each $i$. We call the map $f$ a degeneration. Note that such a map $f$ may not preserve meets. We say that $M$ and $M^{\prime}$ are $l c m$-equivalent if $f$ is an isomorphism.

Remark 4.2. The following are special cases of degeneration:

(1) The ideal $M$ is a reduction of its generic deformations, constructed in 2 , Section 4].

(2) The radical $\operatorname{rad}(M)$ is a reduction of $M$. The degeneration map $f$ maps each monomial to its radical.

(3) The ideal $M$ and its polarization $M_{p o l}$ are lcm-equivalent.

The idea and construction of $f$-degeneration (equivalently, relabeling) is introduced in the paper 8 . We describe degeneration again for the convenience of the reader and because in $[8]$ it is considered only for free resolutions that are minimal. 
Construction 4.3. Let $\mathbf{F}^{\prime}$ be a free multigraded resolution of $S^{\prime} / M^{\prime}$ with a fixed multihomogeneous basis $u_{1}^{\prime}, \ldots, u_{p}^{\prime}$. Let $f\left(\mathbf{F}^{\prime}\right)$ be the free $S$-module with basis denoted $f\left(u_{1}^{\prime}\right), \ldots, f\left(u_{p}^{\prime}\right)$, so that for each $i$ the element $f\left(u_{i}^{\prime}\right)$ has the same homological degree as $u_{i}^{\prime}$, and the multidegree of $f\left(u_{i}^{\prime}\right)$ is $f\left(\operatorname{deg}\left(u_{i}^{\prime}\right)\right)$. We define differential $\partial$ on $f\left(\mathbf{F}^{\prime}\right)$ as follows: for a basis element $u^{\prime}$, if

$$
\partial^{\prime}\left(u^{\prime}\right)=\sum_{1 \leq s \leq q} \alpha_{s j} \frac{\operatorname{deg}\left(u^{\prime}\right)}{\operatorname{deg}\left(u_{s}^{\prime}\right)} u_{s}^{\prime}
$$

with $u_{s}^{\prime}$ elements in the fixed basis, and $\alpha_{s j} \in k$, then we set

$$
\partial\left(f\left(u^{\prime}\right)\right)=\sum_{1 \leq s \leq q} \alpha_{s j} \frac{f\left(\operatorname{deg}\left(u^{\prime}\right)\right)}{f\left(\operatorname{deg}\left(u_{s}^{\prime}\right)\right)} f\left(u_{s}^{\prime}\right) .
$$

We say that $f\left(\mathbf{F}^{\prime}\right)$ is an $f$-degeneration of $\mathbf{F}^{\prime}$. Thus, $f\left(\mathbf{F}^{\prime}\right)$ is multigraded. Straightforward verification shows that $f\left(\mathbf{F}^{\prime}\right)$ is a complex. Note that $\mathbf{F}^{\prime}$ and $f\left(\mathbf{F}^{\prime}\right)$ have the same frame.

The following important property holds by construction:

Lemma 4.4. If $w$ is an element in the fixed basis of $\mathbf{F}^{\prime}$, then the corresponding basis element $f(w)$ in the complex $f\left(\mathbf{F}^{\prime}\right)$ has multidegree $f(\operatorname{deg}(w))$.

Example 4.5. Let $A^{\prime}=k[x, y], N^{\prime}=\left(x^{5}, x^{2} y^{2}, y^{5}\right)$ and $A=k[a, b, c], N=$ $(a b, b c, a c)$. Define $f: L_{N^{\prime}} \rightarrow L_{N}$ by $f\left(x^{5}\right)=a b, f\left(x^{2} y^{2}\right)=b c, f\left(y^{5}\right)=a c, f\left(x^{5} y^{2}\right)$ $=a b c, f\left(x^{2} y^{5}\right)=a b c, f\left(x^{5} y^{5}\right)=a b c$. Then $f$ is a bijection on the atoms and preserves lcm's; however, $f$ is not an isomorphism. The minimal free multigraded resolution $\mathbf{F}_{N^{\prime}}$ of $A^{\prime} / N^{\prime}$ is:

$$
\mathbf{F}_{N^{\prime}}: \quad 0 \rightarrow A^{\prime 2} \stackrel{\left(\begin{array}{cc}
y^{2} & 0 \\
-x^{3} & y^{2} \\
0 & -x^{3}
\end{array}\right)}{\longrightarrow} A^{\prime 3} \stackrel{\left(x^{5}\right.}{\left.\stackrel{x^{2} y^{2}}{y^{5}}\right)} A^{\prime} \rightarrow 0 .
$$

The $f$-degeneration $f\left(\mathbf{F}_{N^{\prime}}\right)$ is:

$$
f\left(\mathbf{F}_{N^{\prime}}\right): 0 \rightarrow A^{2} \stackrel{\left(\begin{array}{cc}
c & 0 \\
-a & a \\
0 & -b
\end{array}\right)}{\longrightarrow} A^{3} \stackrel{\left(\begin{array}{lll}
a b & b c & a c
\end{array}\right)}{\longrightarrow} A \rightarrow 0 .
$$

Theorem 4.6. Let $M$ be a reduction of $M^{\prime}$. Let $\mathbf{F}^{\prime}$ be a free multigraded resolution of $S^{\prime} / M^{\prime}$ with a fixed multihomogeneous basis with degrees in $L_{M^{\prime}}$. The $f$-degeneration $f\left(\mathbf{F}^{\prime}\right)$ is a free multigraded resolution of $S / M$.

Proof. We apply Theorem 3.8 Clearly, $f\left(\mathbf{F}^{\prime}\right)$ is an $M$-complex.

For every monomial $m \in L_{M}$, consider the set of monomials $f^{-1}(m)$ and set

$$
m^{\prime}=\operatorname{lcm}\left\{v^{\prime} \mid v^{\prime} \in f^{-1}(m)\right\} .
$$

Since $f$ preserves lcm's, it follows that $f\left(m^{\prime}\right)=m$. Thus, $m^{\prime}$ is the top (greatest) element in $f^{-1}(m)$. By Lemma 4.4, it follows that $f\left(\mathbf{F}^{\prime}\right)(\leq m)$ and $\mathbf{F}^{\prime}\left(\leq m^{\prime}\right)$ have the same frame. Since $\mathbf{F}^{\prime}$ is exact, by Theorem 3.8 it follows that the frame of $f\left(\mathbf{F}^{\prime}\right)(\leq m)$ is exact. 
Remark 4.7. Theorem 4.6 was proved in the following special cases:

o when $\mathbf{F}^{\prime}$ is the minimal free multigraded resolution of $S^{\prime} / M^{\prime}$ [8, Theorem 3.3];

$\circ$ when $M^{\prime}$ is a generic deformation of $M$ [2, Theorem 4.3].

Note that the proofs of [5. Theorem 7.2] and [8, Theorem 3.3] both use that Taylor's resolution is a direct sum of the minimal free resolution and a trivial complex. So, both proofs work only for a degeneration of a minimal free resolution. Our proof is completely different and works for nonminimal free resolutions as well.

The following result is useful in obtaining bounds for the Betti numbers. It is a straightforward corollary of the previous theorem.

Theorem 4.8. Let $M$ be a reduction of $M^{\prime}$. The total Betti numbers of $S / M$ are smaller than or equal to those of $S^{\prime} / M^{\prime}$.

Remark 4.9. Theorem 4.8 was proved in the special case when $M^{\prime}$ is a generic deformation of $M$ in [2, Corollary 4.4]. We apply Theorem 4.8] in the next section, in order to obtain a lower bound on the Betti numbers in Corollary 7.1 .

Next, we introduce the new construction of $f$-homogenization and then study its relation to $f$-degeneration.

Construction 4.10. Let $M$ be a reduction of $M^{\prime}$. Let $\mathbf{F}^{\prime}$ be a multigraded free resolution of $S^{\prime} / M^{\prime}$ with a fixed multihomogeneous basis. We say that the $M$ homogenization of the frame of $\mathbf{F}^{\prime}$ is an $f$-homogenization of $\mathbf{F}^{\prime}$, and denote it by $\tilde{f}\left(\mathbf{F}^{\prime}\right)$. The role of $f$ in this construction is only to provide an ordering of the minimal monomial generators $m_{1}^{\prime}, \ldots, m_{r}^{\prime}$ of $M^{\prime}$ so that $f\left(m_{i}^{\prime}\right)=m_{i}$ for each $i$.

Example 4.11. This example illustrates that the $f$-degeneration and the $f$ homogenization can differ. Consider the monomial ideal $N^{\prime}=\left(x^{2}, y^{2}, z^{2}\right)$, which is lcm-equivalent to $N=\left(x^{3}, y^{3}, z^{3}\right)$ in the $\operatorname{ring} A=k[x, y, z]$. Let $\mathbf{F}^{\prime}$ be the nonminimal free resolution

$$
\mathbf{F}^{\prime}: 0 \rightarrow A^{2} \stackrel{\left.\left(\begin{array}{cc}
z^{2} & -z^{2} \\
x^{2} & 0 \\
-y^{2} & 0 \\
0 & 1
\end{array}\right)^{4}\left(\begin{array}{cccc}
y^{2} & 0 & z^{2} & y^{2} z^{2} \\
-x^{2} & z^{2} & 0 & -x^{2} z^{2} \\
0 & -y^{2} & -x^{2} & 0
\end{array}\right) \text { } A^{3} \stackrel{\left(x^{2}\right.}{\longrightarrow} y^{2} z^{2}\right)}{\longrightarrow} A \rightarrow 0 .
$$

Its frame is

$$
0 \rightarrow k^{2} \stackrel{\left(\begin{array}{cc}
1 & -1 \\
1 & 0 \\
-1 & 0 \\
0 & 1
\end{array}\right)}{\longrightarrow} k^{4} \stackrel{\left(\begin{array}{cccc}
1 & 0 & 1 & 1 \\
-1 & 1 & 0 & -1 \\
0 & -1 & -1 & 0
\end{array}\right)}{\longrightarrow} k^{3} \stackrel{\left(\begin{array}{lll}
1 & 1 & 1
\end{array}\right)}{\longrightarrow} k \rightarrow 0 .
$$

Therefore, the $f$-homogenization is

$\tilde{f}\left(\mathbf{F}^{\prime}\right): 0 \rightarrow A^{2} \stackrel{\left(\begin{array}{cc}z^{3} & -1 \\ x^{3} & 0 \\ -y^{3} & 0 \\ 0 & 1\end{array}\right)}{\longrightarrow} A^{4} \stackrel{\left(\begin{array}{cccc}y^{3} & 0 & z^{3} & y^{3} \\ -x^{3} & z^{3} & 0 & -x^{3} \\ 0 & -y^{3} & -x^{3} & 0\end{array}\right)}{\longrightarrow} A^{3} \stackrel{\left(x^{3}\right.}{\left.\stackrel{y^{3}}{z^{3}}\right)} \rightarrow A \rightarrow 0$. 
On the other hand, the $f$-degeneration is

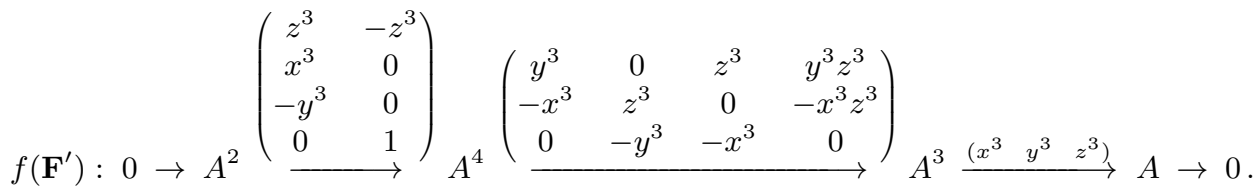

From Constructions 4.3 and 4.10 we see that:

Lemma 4.12. Let $M$ be a reduction of $M^{\prime}$. Let $\mathbf{F}^{\prime}$ be a multigraded free resolution of $S^{\prime} / M^{\prime}$ with a fixed multihomogeneous basis with degrees in $L_{M^{\prime}}$. The $f$-homogenization and the $f$-degeneration of $\mathbf{F}^{\prime}$ coincide if and only if the following property $\left({ }^{*}\right)$ is satisfied: for every $w$ in the fixed basis we have that $\operatorname{deg}(w)=$ $\operatorname{lcm}\left(\operatorname{deg}\left(u_{s}\right) \mid m_{s j} \neq 0\right)$, where $\partial(w)=\sum_{1 \leq s \leq q} m_{s j} u_{s}$ with $u_{s}$ elements in the fixed basis (note that each $m_{s j}$ is a monomial multiplied by a scalar).

Theorem 4.13. Let $M$ be a reduction of $M^{\prime}$. Let $\mathbf{F}^{\prime}$ be a minimal free multigraded resolution of $S^{\prime} / M^{\prime}$ with a fixed multihomogeneous basis.

(1) The $f$-homogenization $\tilde{f}\left(\mathbf{F}^{\prime}\right)$ and the $f$-degeneration $f\left(\mathbf{F}^{\prime}\right)$ coincide and are the same free resolution of $S / M$.

(2) The free resolution $f\left(\mathbf{F}^{\prime}\right)=\tilde{f}\left(\mathbf{F}^{\prime}\right)$ does not depend on the choice of basis.

(3) If $S / M$ and $S^{\prime} / M^{\prime}$ have the same total Betti numbers, then the free resolution $f\left(\mathbf{F}^{\prime}\right)=\tilde{f}\left(\mathbf{F}^{\prime}\right)$ is minimal.

(4) If $f$ is an isomorphism, then the free resolution $f\left(\mathbf{F}^{\prime}\right)=\tilde{f}\left(\mathbf{F}^{\prime}\right)$ is minimal.

Proof. First, we will prove (1). We will show that Lemma4.12 can be applied. Let $w$ be a multihomogeneous element in some multihomogeneous basis of $\mathbf{F}^{\prime}$ and let

$$
\partial^{\prime}(w)=\sum_{1 \leq s \leq q} a_{j s} u_{s}
$$

where the $u_{s}$ are multihomogeneous basis elements; note that each $a_{j s}$ is a monomial multiplied by a scalar. Since $\mathbf{F}^{\prime}$ is minimal, at least one of the coefficients $a_{j s}$ does not vanish. We will prove that $\operatorname{deg}(w)=\operatorname{lcm}\left(\operatorname{deg}\left(u_{s}\right) \mid a_{j s} \neq 0\right)$. Assume the opposite. Therefore, there exists a monomial $b \neq 1$ such that

$$
\partial^{\prime}(w)=b \sum_{1 \leq s \leq q} \tilde{a}_{j s} u_{s},
$$

where $b \tilde{a}_{j s}=a_{j s}$. Since $\partial^{\prime 2}(w)=0$, it follows that $\partial^{\prime 2}\left(\sum_{1 \leq s \leq q} \tilde{a}_{j s} u_{s}\right)=0$. Therefore, $\partial^{\prime}(w) \in b \operatorname{Ker}\left(\partial^{\prime}\right)$. By Nakayama's Lemma, it follows that $\partial^{\prime}(w)$ is not an element in any multihomogeneous minimal system of generators of the kernel. On the other hand, $\partial^{\prime}(w)$ is an element in a multihomogeneous minimal system of generators of the kernel because $\mathbf{F}^{\prime}$ is minimal. This is a contradiction.

Thus, $\operatorname{deg}(w)=\operatorname{lcm}\left(\operatorname{deg}\left(u_{s}\right) \mid a_{j s} \neq 0\right)$.

By Lemma 4.12, it follows that the $f$-homogenization $\tilde{f}\left(\mathbf{F}^{\prime}\right)$ and the $f$-degeneration $f\left(\mathbf{F}^{\prime}\right)$ coincide. By Theorem 4.6, $f\left(\mathbf{F}^{\prime}\right)$ is a free multigraded resolution of $S / M$. (2) holds since the multidegrees of the basis elements in $\mathbf{F}^{\prime}$ are the same in any choice of basis; they are determined by the multigraded Betti numbers. (3) is clear. Furthermore, (4) follows from (3) and Theorem 2.2.

The following important result is a straightforward corollary of the above theorem. 
Theorem 4.14. The $M$-homogenization of any frame of the minimal multigraded free resolution $\mathbf{F}$ of $S / M$ is $\mathbf{F}$.

Corollary 4.15. Let $M$ be a reduction of $M^{\prime}$. Suppose that $S / M$ and $S^{\prime} / M^{\prime}$ have the same total Betti numbers. There exists a multihomogeneous basis of the minimal free multigraded resolution $\mathbf{F}$ of $S / M$, such that the $M^{\prime}$-homogenization of the frame of $\mathbf{F}$ is a minimal free multigraded resolution of $S^{\prime} / M^{\prime}$.

Proof. Let $\mathbf{F}^{\prime}$ be a minimal free multigraded resolution of $S^{\prime} / M^{\prime}$ with a fixed multihomogeneous basis $\mathcal{F}^{\prime}$. By Theorem 4.13 the $M$-homogenization of the frame of $\mathbf{F}^{\prime}$ is $\mathbf{F}$ with a fixed multihomogeneous basis, which we denote by $\mathcal{F}$. Thus, in the bases $\mathcal{F}^{\prime}$ and $\mathcal{F}$, the frames of $\mathbf{F}^{\prime}$ and $\mathbf{F}$ coincide. Since the resolution $\mathbf{F}^{\prime}$ is minimal, by Corollary 4.14 we have that the $M^{\prime}$-homogenization of the frame of $\mathbf{F}^{\prime}$ is $\mathbf{F}^{\prime}$. Therefore, the $M^{\prime}$-homogenization of the frame of $\mathbf{F}$ is $\mathbf{F}^{\prime}$.

One may ask if Corollary 4.15 does not depend on the choice of basis, that is, if the $M^{\prime}$-homogenization of any frame of $\mathbf{F}$ is a minimal multigraded free resolution of $S^{\prime} / M^{\prime}$. The answer is negative by Example 7.3 .

It is worth noting the following result, which is straightforward from the constructions of cellular resolutions and Taylor's resolution.

Proposition 4.16. Let $M$ be a reduction of $M^{\prime}$.

(1) If $\mathbf{F}^{\prime}$ is a cellular free resolution of $S^{\prime} / M^{\prime}$, then the $f$-homogenization and the $f$-degeneration of $\mathbf{F}^{\prime}$ coincide.

(2) Let $\mathbf{T}^{\prime}$ be Taylor's resolution of $S^{\prime} / M^{\prime}$ (with the basis used in the construction of Taylor's resolution). The $f$-degeneration and the $f$-homogenization of $\mathbf{T}^{\prime}$ coincide and are the Taylor's resolution of $S / M$.

(3) Suppose that $M$ and $M^{\prime}$ are generated by regular monomial sequences. Let $\mathbf{K}^{\prime}$ be the Koszul resolution of $S^{\prime} / M^{\prime}$ (with the basis used in the construction of the Koszul complex). The $f$-degeneration and the $f$-homogenization of $\mathbf{K}^{\prime}$ coincide and are the Koszul resolution of $S / M$.

\section{SCARF COMPlexes}

Construction 5.1. For each subset $\tau$ of $\{1, \ldots, r\}$ we set $m_{\tau}=\operatorname{lcm}\left(m_{i} \mid i \in \tau\right)$; by convention, $\operatorname{lcm}(\emptyset)=1$. The Scarf complex of $M$ is the simplicial complex

$$
\Omega_{M}=\left\{\tau \subseteq\{1, \ldots, r\} \mid m_{\tau} \neq m_{\sigma} \text { for all } \sigma \subseteq\{1, \ldots, r\} \text { other than } \tau\right\} .
$$

In 2 it is shown that $\Omega_{M}$ coincides with a simplicial complex introduced by Scarf in the context of mathematical economics. Denote by $\mathbf{F}_{\Omega_{M}}$ the $M$-homogenization of the augmented oriented simplicial chain complex of $\Omega_{M}$. Following [2], we call $M$ a Scarf $\underline{\text { ideal }}$ if $\mathbf{F}_{\Omega_{M}}$ is the minimal free resolution of $S / M$, and we say that the complex $\overline{\mathbf{F}_{\Omega_{M}}}$ is its $\underline{\text { Scarf }} \underline{\text { resolution }}$. In this case we say that $\Omega_{M}$ supports a Scarf resolution.

The multidegree of a vertex $v_{i}$ in $\Omega_{M}$ is $m_{i}$. The multidegree of a face $\tau \in \Omega_{M}$ is $\operatorname{deg}(\tau)=\operatorname{lcm}(\operatorname{deg}(v) \mid v$ is a vertex of $\tau)$; this is the multidegree of the basis element $\tau$ in $\mathbf{F}_{\Omega_{M}}$. The multidegrees of the faces of $\Omega_{M}$ are called the Scarf multidegrees .

Example 5.2. The Scarf complex of $L=\left(x^{3}, x y, y^{5}\right)$ has three vertices $x^{3}, x y, y^{5}$ and the two edges $\left\{x^{3}, x y\right\},\left\{x y, y^{5}\right\}$. 


\section{Theorem 5.3.}

(1) A finite simplicial complex with $r$ vertices is the Scarf complex of a monomial ideal if and only if it is not the boundary of the simplex with $r$ vertices.

(2) A finite simplicial complex $\Omega$ supports a Scarf resolution if and only if $\Omega$ is acyclic.

Proof. Let $\Omega$ be a finite simplicial complex. If $\Omega$ is a point or $\emptyset$, then (1) and (2) hold. Assume that $\Omega$ has at least two vertices.

(1) For each face $\tau$ of $\Omega$ introduce a variable $x_{\tau}$. Consider the polynomial ring $B=k\left[x_{\tau} \mid \tau \in \Omega, \tau \neq \emptyset\right]$. Set the multidegree of each vertex $v$ of $\Omega$ to be $\operatorname{deg}(v)=\prod_{v \notin \tau \in \Omega} x_{\tau}$. It follows that a face $\sigma$ has multidegree

$$
\operatorname{deg}(\sigma)=\operatorname{lcm}(\operatorname{deg}(v) \mid v \in \sigma)=\prod_{\sigma \nsubseteq \tau \in \Omega} x_{\tau} .
$$

Therefore, every two faces have distinct multidegrees. Let $\Theta$ be the simplex on the vertices of $\Omega$. If $\mu$ is a face of $\Theta$ and $\mu \notin \Omega$, then $\mu$ has multidegree $z$, where $z$ is the product of all the variables. Let $J_{\Omega}$ be the ideal generated by the multidegrees of the vertices. The complex $\Omega$ has at least two nonfaces if and only if it is not the boundary of $\Theta$. Therefore, $\Omega$ is the Scarf complex of the ideal $J_{\Omega}$ if and only if $\Omega$ is not the boundary of $\Theta$.

(2) If $\Omega$ supports a Scarf resolution, then it is acyclic by Theorem 3.8 applied to the multidegree $m$ that is the $\mathrm{lcm}$ of all the minimal monomial generators of the ideal. Now, suppose that $\Omega$ is acyclic. We will show that the ideal $J_{\Omega}$ constructed in (1) is a Scarf ideal. The lcm of its minimal monomial generators is $z$. The lcm-lattice consists of Scarf multidegrees (including the bottom element 1) and the top element $z$. By Theorem 6.1 (proved later), we conclude that $\Omega$ supports the Scarf resolution of $J_{\Omega}$.

As a corollary, we characterize the possible sequences of Betti numbers of Scarf ideals:

Corollary 5.4. Let $b_{0}=1, b_{1}, b_{2}, \ldots$ be a finite sequence of natural numbers. For each $i \geq 0$, set $\alpha_{i}=\sum_{j \geq 0}(-1)^{j} b_{i+j}$. The sequence $b_{1}, b_{2}, \ldots$ is the sequence of total Betti numbers of a Scarf ideal if and only the sequence $\alpha_{0}, \alpha_{1}, \ldots$ is the Hilbert function of the quotient $k\left[x_{1}, \ldots, x_{\alpha_{1}}\right] /\left(T+\left(x_{1}^{2}, \ldots, x_{\alpha_{1}}^{2}\right)\right)$ for some squarefree monomial ideal $T$.

Proof. By Theorem 5.3, it follows that $b_{1}, b_{2}, \ldots$ is the sequence of Betti numbers of a Scarf ideal if and only if $b_{0}=1, b_{1}, b_{2}, \ldots$ is the $f$-vector of an acyclic complex $\Omega$, that is, $b_{i}$ is the number of $i$-dimensional faces of $\Omega$. The $f$-vectors of acyclic simplicial complexes are characterized in [12].

\section{NeARly ScARF IDEALS}

Throughout this section, $\Omega$ is a finite simplicial complex with at least 2 vertices. We say that the ideal $J_{\Omega}$, constructed in the proof of Theorem 5.3.1), is the nearly-Scarf ideal of $\Omega$. It is a squarefree ideal in the polynomial ring $\left.B=\overline{k\left[x_{\tau} \mid \tau \in \Omega\right.}, \tau \neq \emptyset\right]$. If $\Omega$ is acyclic, then $J_{\Omega}$ is a Scarf ideal. We use the notation introduced in the previous section. 
Theorem 6.1. Let $J$ be a monomial ideal in $S$ whose lcm-lattice consists of the Scarf multidegrees (including the bottom element 1 ) and a top element $y$. Let $\Omega$ be the Scarf complex of $J$ and let

$$
\mathbf{C}: \quad 0 \rightarrow C_{\operatorname{dim}(\Omega)}(\Omega, k) \rightarrow \cdots \rightarrow C_{0}(\Omega, k) \rightarrow C_{-1}(\Omega, k) \rightarrow 0
$$

be the oriented augmented homology chain complex of $\Omega$ with differential $\partial$. For each $i$, choose a set $\left\{q_{1}, \ldots, q_{p}\right\}$ of cycles whose classes in $\operatorname{Ker}\left(\partial_{i}\right) / \operatorname{Im}\left(\partial_{i+1}\right)$ form $a$ basis and set

$$
\begin{aligned}
\phi_{i}: k^{\operatorname{dim}\left(\tilde{\mathrm{H}}_{i}(\Omega, k)\right)} & \rightarrow \operatorname{Ker}\left(\partial_{i}\right), \\
e_{j} & \mapsto q_{j},
\end{aligned}
$$

where $e_{1}, \ldots$ are the standard basis elements. Let $\mathbf{U}$ be the complex

$\mathbf{U}: 0 \rightarrow k^{\operatorname{dim} \tilde{H}_{\operatorname{dim}(\Omega)}(\Omega, k)} \rightarrow \cdots \rightarrow C_{i}(\Omega, k) \oplus k^{\operatorname{dim}_{i-1}(\Omega, k)} \rightarrow \cdots \rightarrow C_{-1}(\Omega, k) \rightarrow 0$

with differential $\partial \oplus \phi$. The J-homogenization of the complex $\mathbf{U}$ is the multigraded minimal free resolution of $S / J$.

Proof. Let $\sigma_{1}+\cdots+\sigma_{p}$, where $\sigma_{j} \in \Omega$, be a cycle that is nontrivial in the homology $\tilde{\mathrm{H}}_{i}(\Omega, k)$. We will prove that $\operatorname{lcm}\left(\operatorname{deg}\left(\sigma_{j}\right) \mid 1 \leq j \leq p\right)=y$. Assume the opposite. It follows that $\operatorname{lcm}\left(\operatorname{deg}\left(\sigma_{j}\right) \mid 1 \leq j \leq p\right)=\operatorname{deg}(\tau)$ for some $\tau \in \Omega$. By the definition of the Scarf complex, it follows that all the faces $\sigma_{1}, \ldots, \sigma_{p}$ are subfaces of $\tau$. But $\tau$ is a simplex, contradicting the fact that $\sigma_{1}+\cdots+\sigma_{p}$ is nontrivial in homology. Thus, $\operatorname{lcm}\left(\operatorname{deg}\left(\sigma_{j}\right) \mid 1 \leq j \leq p\right)=y$.

Denote by $\mathbf{G}$ the $J$-homogenization of the complex $\mathbf{U}$. We will apply Theorem 3.8 in order to show that $\mathbf{G}$ is exact. Let $m \in L_{J}$. First, suppose that $m=y$. Then the frame of $\mathbf{G}(\leq m)$ is the complex $\mathbf{U}$, which is exact. Now, suppose that $m=\operatorname{deg}(\tau)$ for some $\tau \in \Omega$. Then the frame of $\mathbf{G}(\leq m)$ is the oriented augmented homology chain complex of the simplex $\tau$, so it is exact.

Corollary 6.2. The ideals $J$ and $J_{\Omega}$ have isomorphic lcm-lattices. The $J_{\Omega}$ homogenization of the complex $\mathbf{U}$ (in Theorem 6.1) is the multigraded minimal free resolution of $B / J_{\Omega}$.

Proof. Set $z$ to be the product of all the variables in the $\operatorname{ring} B=k\left[x_{\tau} \mid \tau \in \Omega, \tau \neq\right.$ $\emptyset]$. Recall that the lcm-lattice of $J_{\Omega}$ consists of the Scarf multidegrees (including the bottom element 1) and the top element $z$.

We denote by $|\Omega|$ the number of nonempty faces of the complex $\Omega$; it is equal to the degree of the monomial $z$. For a face $\sigma \in \Omega$, the degree of the monomial $\operatorname{deg}(\sigma)=\prod_{\{\tau \in \Omega \mid \sigma \nsubseteq \tau\}} x_{\tau}$ is equal to the number $|\{\tau \in \Omega \mid \sigma \nsubseteq \tau\}|$. Furthermore, let

$$
\tilde{\chi}(\Omega)=\sum_{i=-1}^{\operatorname{dim}(\Omega)}(-1)^{i} \operatorname{dim} \tilde{H}_{i}(\Omega, k)
$$

be the reduced Euler characteristic of $\Omega$.

Corollary 6.3. The Hilbert series of $B / J_{\Omega}$ is

$$
\frac{\tilde{\chi}(\Omega) t^{|\Omega|}-\sum_{\sigma \in \Omega}(-1)^{\operatorname{dim}(\sigma)} t^{|\{\tau \in \Omega \mid \sigma \not \tau\}|}}{(1-t)^{|\Omega|}} .
$$


Furthermore,

$$
\begin{aligned}
\operatorname{pd}\left(B / J_{\Omega}\right) & = \begin{cases}\operatorname{dim}(\Omega)+1 & \text { if } \tilde{\mathrm{H}}_{\operatorname{dim}(\Omega)}(\Omega, k)=0, \\
\operatorname{dim}(\Omega)+2 & \text { otherwise },\end{cases} \\
\operatorname{codim}\left(J_{\Omega}\right) & =2, \\
\operatorname{reg}\left(J_{\Omega}\right) & =\max \left\{|\Omega|-\min \left\{i \mid \tilde{\mathrm{H}}_{i-2}(\Omega, k) \neq 0\right\},\right. \\
& \max \{|\{\tau \in \Omega \mid \sigma \not \subset \tau\}|-\operatorname{dim}(\sigma)-1 \mid \sigma \in \Omega\}\} .
\end{aligned}
$$

Proof. The formulas for the Hilbert series and the projective dimension follow from Corollary 6.2. The formula for the regularity holds because

$$
\begin{aligned}
& \operatorname{reg}\left(J_{\Omega}\right)=\max \left\{\max \left\{|\Omega|-p \mid b_{p, z}\left(S / J_{\Omega}\right) \neq 0\right\},\right. \\
&\left.\max \left\{(\text { the degree of } \operatorname{deg}(\sigma))-p \mid b_{p, \operatorname{deg}(\sigma)}\left(S / J_{\Omega}\right) \neq 0, \sigma \in \Omega\right\}\right\} \max \left\{|\Omega|-i \mid \tilde{\mathrm{H}}_{i-2}(\Omega, k) \neq 0\right\}, \\
&\max \{|\{\tau \in \Omega \mid \sigma \not \subset \tau\}|-\operatorname{dim}(\sigma)-1 \mid \sigma \in \Omega\}\} .
\end{aligned}
$$

It remains to compute the codimension. The ideal $J_{\Omega}$ is squarefree, so it is the Stanley-Reisner ideal of a simplicial complex $\Delta$ on the vertex set $\{\emptyset \neq \tau \in \Omega\}$. We have that $\operatorname{dim}\left(B / J_{\Omega}\right)=\operatorname{dim}(\Delta)+1$. The ring $B$ has $|\Omega|$ variables, so $\operatorname{codim}\left(J_{\Omega}\right)=$ $|\Omega|-\operatorname{dim}(\Delta)-1$.

By the definition of $J_{\Omega}$ it follows that $\left\{\tau_{1}, \ldots, \tau_{i}\right\} \in \Delta$ if and only if for each $\emptyset \neq \tau \in \Omega$ there exists a $\sigma \in \Omega$ such that $\sigma \nsupseteq \tau$ and $\sigma \notin\left\{\tau_{1}, \ldots, \tau_{i}\right\}$. Therefore, $\Delta$ has no faces of dimension $|\Omega|-2$. Also, for every two disjoint nonempty $\tau_{1}, \tau_{2} \in \Omega$, we have that $\{\emptyset \neq \tau \in \Omega\} \backslash\left\{\tau_{1}, \tau_{2}\right\}$ is a face in $\Delta$. Hence, $\operatorname{dim}(\Delta)=|\Omega|-3$.

Corollary 6.4. The ring $S / J_{\Omega}$ is Cohen-Macaulay if and only if $\Omega$ is a forest.

Proof. By the Auslander-Buchsbaum formula, $S / J_{\Omega}$ is Cohen-Macaulay if and only if $\operatorname{pd}\left(S / J_{\Omega}\right)=\operatorname{codim}\left(J_{\Omega}\right)=2$. This happens if and only if either $\operatorname{dim}(\Omega)=0$ and $\tilde{\mathrm{H}}_{0}(\Omega, k) \neq 0$, or $\operatorname{dim}(\Omega)=1$ and $\tilde{\mathrm{H}}_{1}(\Omega, k)=0$. In the former case, $\Omega$ is a set of points; in the latter case, $\Omega$ is a collection of trees.

\section{Monomial ideals with smallest Betti numbers}

Using nearly-Scarf ideals, we obtain a lower bound for the Betti numbers of a monomial ideal. Furthermore, we describe the structure of the minimal free resolution for a monomial ideal with minimal Betti numbers among all monomial ideals with a fixed Scarf complex.

Let $\Omega$ be the Scarf complex of a monomial ideal $M$. For a chain $q$ in $\Omega$, we define $\operatorname{deg}(q)$ to be the $\mathrm{lcm}$ of the degrees of the faces in its support. For a monomial $m \in L_{M}$, we define the subcomplex $\Omega_{\preceq m}=\{\tau \in \Omega \mid \operatorname{deg}(\tau)$ divides $m\}$ of $\Omega$.

Theorem 7.1. Let $\Omega$ be the Scarf complex of a monomial ideal $M$. Denote by $f_{i}(\Omega)$ the number of $i$-dimensional faces of $\Omega$. 
(1) We have that

$$
b_{i}(S / M) \geq f_{i-1}(\Omega)+\operatorname{dim} \widetilde{\mathrm{H}}_{i-2}(\Omega ; k) .
$$

(2) Suppose that for each $i$,

$$
b_{i}(S / M)=f_{i-1}(\Omega)+\operatorname{dim} \widetilde{\mathrm{H}}_{i-2}(\Omega ; k) .
$$

Then there exists a basis of $\widetilde{\mathrm{H}}_{*}(\Omega ; k)$ which satisfies the following property:

$$
\begin{aligned}
& \text { for every multidegree } m \in L_{M} \text {, } \\
& \text { the elements whose degrees divide } m \text { form a basis of } \widetilde{\mathrm{H}}_{*}\left(\Omega_{\preceq m} ; k\right) \text {. }
\end{aligned}
$$

Moreover, using any basis that satisfies (7.2) as the choice of cycles in Theorem 6.1, the $M$-homogenization of the frame $\mathbf{U}$ is the minimal free resolution of $S / M$.

Proof. First, we will prove (1). We use the notation and the construction in the proof of Theorem $5.3(1)$. Let $z$ be the product of all the variables in the polynomial ring $B=k\left[x_{\tau} \mid \tau \in \Omega, \tau \neq \emptyset\right]$. Consider the map $f: L_{M} \rightarrow L_{J_{\Omega}}$ that preserves each monomial that is the multidegree of a face in $\Omega$ and maps all other monomials to $z$. This map is a bijection on the atoms and preserves lcm's. Hence, the nearly-Scarf ideal $J_{\Omega}$ is a reduction of the ideal $M$. By Theorem 4.8 ,

$$
b_{i}(S / M) \geq b_{i}\left(B / J_{\Omega}\right)
$$

for all $i$.

We will compute the Betti number $b_{i}\left(B / J_{\Omega}\right)$. By Theorem 2.2 we get

$$
b_{i, z}\left(B / J_{\Omega}\right)=\operatorname{dim} \widetilde{\mathrm{H}}_{i-2}((1, z) ; k)=\operatorname{dim} \widetilde{\mathrm{H}}_{i-2}(\Omega ; k),
$$

since the order complex of the open interval $(1, z)$ (in $L_{J_{\Omega}}$ ) and $\Omega$ are homotopic. By Theorem 2.2 we also get

$$
\sum_{u \in L_{J_{\Omega}}, u \neq z} b_{i, u}\left(B / J_{\Omega}\right)=f_{i-1}(\Omega) .
$$

Therefore,

$$
\begin{aligned}
b_{i}\left(B / J_{\Omega}\right) & =b_{i, z}\left(B / J_{\Omega}\right)+\sum_{u \in L_{J_{\Omega}}, u \neq z} b_{i, u}\left(B / J_{\Omega}\right) \\
& =f_{i-1}(\Omega)+\operatorname{dim} \widetilde{\mathrm{H}}_{i-2}(\Omega ; k) .
\end{aligned}
$$

Thus, (1) is proved.

We will prove (2). We use the notation introduced in Theorem 6.1 Denote by $\mathbf{F}_{M}$ the minimal free resolution of $S / M$ over $S$.

First, we will show that there exists a basis with the desired properties. The $M$-homogenization $\mathbf{C}_{M}$ of the frame $\mathbf{C}$ is the algebraic Scarf complex of $M$. Fix a basis $\mathcal{F}$ of $\mathbf{F}_{M}$ that contains the basis $\mathcal{C}$ of $\mathbf{C}_{M}$. We can write $\mathcal{F}=\mathcal{C} \cup \mathcal{V}$ as a disjoint union. The number of elements in the set $\mathcal{F}_{i}$ is $b_{i}(S / M)=f_{i-1}(\Omega)+\operatorname{dim} \widetilde{\mathrm{H}}_{i-2}(\Omega ; k)$. The number of elements in the set $\mathcal{C}_{i}$ is $f_{i-1}(\Omega)$. Therefore, the number of elements in the set $\mathcal{V}_{i}$ is $\operatorname{dim} \widetilde{\mathrm{H}}_{i-2}(\Omega ; k)$.

Consider the frame $\mathbf{U}$ of $\mathbf{F}_{M}$; denote its differential by $d$ and its restriction to the subcomplex $\mathbf{C}$ by $\partial$. Since $\mathbf{F}_{M}$ is a free resolution, by Theorem 3.8 (2) it follows that $\mathbf{U}$ is exact.

We will show by induction on $i \geq 1$ that $d_{i}\left(\mathcal{V}_{i}\right) \subseteq \operatorname{span}\left(\mathcal{C}_{i-1}\right)$ is a set of cycles whose images in $\widetilde{\mathrm{H}}_{i-2}(\Omega ; k)$ form a basis. For $i=1$ the statement is obvious 
since $d_{1}=\partial_{0}$. Let $i>1$. The induction hypothesis guarantees that $\operatorname{Ker}\left(d_{i-1}\right)=$ $\operatorname{Ker}\left(\partial_{i-2}\right)$ since for any $v \in \operatorname{span}\left(\mathcal{V}_{i-1}\right)$ and $c \in \operatorname{span}\left(\mathcal{C}_{i-1}\right)$ the equality $0=d_{i-1}(v+$ $c)=d_{i-1}(v)+\partial_{i-2}(c)$ implies that $v=0$. Since $\mathbf{U}$ is exact, the vectors $d_{i}\left(\mathcal{V}_{i}\right) \cup d_{i}\left(\mathcal{C}_{i}\right)$ span $\operatorname{Ker}\left(d_{i-1}\right)=\operatorname{Ker}\left(\partial_{i-2}\right)$, so the cycles $d_{i}\left(\mathcal{V}_{i}\right)$ generate $\operatorname{Ker}\left(\partial_{i-2}\right) / \operatorname{Im}\left(\partial_{i-1}\right)$. Since the number of elements in $\mathcal{V}_{i}$ is $\operatorname{dim} \widetilde{H}_{i-2}(\Omega, k)$, it follows that their classes form a basis.

Thus, $\mathcal{F}=\mathcal{C} \cup \mathcal{V}$ and $\mathcal{V}$ is a basis of $\widetilde{\mathrm{H}}_{*}(\Omega ; k)$. Let $m \in L_{M}$. By Theorem 3.8(2), the frame of $\mathbf{F}_{M}(\leq m)$ is exact. By Construction 3.7 the basis of this frame consists of the elements in $\mathcal{F}$ whose degrees divide $m$. Since $\mathbf{C}_{M}(\leq m)$ is the oriented augmented homology chain complex of $\Omega_{\preceq m}$, it follows that the elements in $\mathcal{V}$ whose degrees divide $m$ form a basis of $\widetilde{\mathrm{H}}_{*}\left(\Omega_{\preceq m} ; k\right)$.

We have shown that every multihomogeneous basis of $\mathbf{F}_{M}$ that contains $\mathcal{C}$ satisfies condition (7.2).

Now, let $\mathcal{V}$ be a basis of $\widetilde{\mathrm{H}}_{*}(\Omega ; k)$ that satisfies condition (7.2). We will show that the $M$-homogenization $\mathbf{G}$ of the frame $\mathbf{U}$ is the minimal free resolution of $S / M$. For every $m \in L_{M}$, the frame of $\mathbf{G}(\leq m)$ is

$$
\begin{aligned}
0 & \rightarrow k^{\operatorname{dim} \tilde{H}_{\operatorname{dim}\left(\Omega_{\preceq m}\right)}\left(\Omega_{\preceq m}, k\right)} \rightarrow \ldots \\
\ldots & \rightarrow C_{i}\left(\Omega_{\preceq m}, k\right) \oplus k^{\operatorname{dim} \tilde{H}_{i-1}\left(\Omega_{\preceq m}, k\right)} \rightarrow \cdots \rightarrow C_{-1}\left(\Omega_{\preceq m}, k\right) \rightarrow 0,
\end{aligned}
$$

so it is exact. By Theorem $3.8(2)$, it follows that $\mathbf{G}$ is exact. Therefore, $\mathbf{G}$ is a multigraded free resolution of $S / M$. Since the ranks of the free modules in $\mathbf{G}$ coincide with the Betti numbers, we conclude that the resolution is minimal.

Remark 7.2. The weaker lower bound $b_{i}(S / M) \geq f_{i-1}(\Omega)$ is proved in 2, remark before Lemma 3.1].

Example 7.3. This example shows that Corollary 4.15] and Theorem[7.1(2) depend on the choice of basis. We construct two ideals $M$ and $M^{\prime}$ such that:

$\circ M$ is a reduction of $M^{\prime}$,

$\circ M$ is nearly Scarf,

- $M$ and $M^{\prime}$ have the same Scarf complex,

- $M$ and $M^{\prime}$ have the same total Betti numbers,

- both $M$ and $M^{\prime}$ satisfy equalities in Theorem 7.1(1),

but there exists a multihomogeneous basis of the minimal free resolution $\mathbf{F}$ of $S / M$ so that the $M^{\prime}$-homogenization of $\mathbf{F}$ is not exact, so is not a free resolution of $S^{\prime} / M^{\prime}$.

Let $\Omega$ be the simplicial complex on 6 vertices that consists of two empty triangles and an edge with a common vertex: denote by $a, \ldots, f$ the vertices and

$$
\begin{aligned}
& j_{1}=\{a, b\}, j_{2}=\{b, c\}, j_{3}=\{a, c\}, j_{4}=\{a, d\}, \\
& j_{5}=\{d, e\}, j_{6}=\{a, e\}, j_{7}=\{a, f\}
\end{aligned}
$$

the edges. Let $M$ be the nearly Scarf ideal $J_{\Omega}$. Also, consider the ideal

$$
M^{\prime}=(\operatorname{deg}(a), \operatorname{deg}(b), \operatorname{deg}(c), x \operatorname{deg}(d), x \operatorname{deg}(e), \operatorname{deg}(f)) .
$$

We consider both ideals in the polynomial ring $V=k\left[a, \ldots, f, j_{1}, \ldots, j_{7}, x\right]$. The monomial $z=a \ldots f j_{1} \ldots j_{7}$ is the top element in $L_{M}$. The lcm-lattice $L_{M^{\prime}}$ consists of $L_{M}$ and the monomial $x z$. The two ideals have the same Scarf complex and the same total Betti numbers. However, $2=b_{3}^{V}(V / M)=b_{3, z}^{V}(V / M)$ is in one 
multidegree, whereas $2=b_{3}^{V}\left(V / M^{\prime}\right)$ is in two different multidegrees $b_{3, z}^{V}\left(V / M^{\prime}\right)=$ $b_{3, x z}^{V}\left(V / M^{\prime}\right)=1$.

Using the chains

$$
q_{1}=j_{1}+j_{2}-j_{3}+j_{4}+j_{5}-j_{6} \quad \text { and } \quad q_{2}=j_{4}+j_{5}-j_{6},
$$

which form a basis of $\widetilde{\mathrm{H}}_{*}(\Omega ; k)$, we obtain the following complex $\mathbf{U}$ as in Theorem 6.1

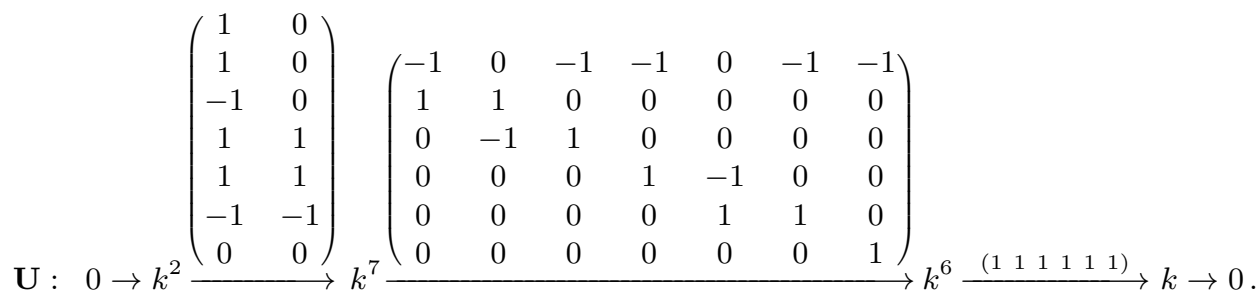

By Theorem 6.1 the $M$-homogenization of $\mathbf{U}$ is the minimal free resolution $\mathbf{F}$ of $V / M$. However, the $M^{\prime}$-homogenization $\mathbf{G}$ of $\mathbf{U}$ is not a minimal free resolution of $V / M^{\prime}$ since it is not exact by Theorem $3.8(2)$ : the frame of $\mathbf{G}(\leq z)$ is isomorphic to the reduced homology chain complex of the subcomplex of $\Omega$ supported on the vertices $a, b, c, f$, which is not acyclic.

On the other hand, using the chains

$$
p_{1}=j_{1}+j_{2}-j_{3}+j_{4}+j_{5}-j_{6} \quad \text { and } \quad p_{2}=j_{1}+j_{2}-j_{3},
$$

which form a basis of $\widetilde{\mathrm{H}}_{*}(\Omega ; k)$, we obtain the following complex $\mathbf{U}^{\prime}$ as in Theorem 6.1 .

$\mathbf{U}^{\prime}: \quad 0 \rightarrow k^{2} \stackrel{\left(\begin{array}{cc}1 & 1 \\ 1 & 1 \\ -1 & -1 \\ 1 & 0 \\ 1 & 0 \\ -1 & 0 \\ 0 & 0\end{array}\right)}{\longrightarrow} k^{7} \stackrel{\left(\begin{array}{ccccccc}-1 & 0 & -1 & -1 & 0 & -1 & -1 \\ 1 & 1 & 0 & 0 & 0 & 0 & 0 \\ 0 & -1 & 1 & 0 & 0 & 0 & 0 \\ 0 & 0 & 0 & 1 & -1 & 0 & 0 \\ 0 & 0 & 0 & 0 & 1 & 1 & 0 \\ 0 & 0 & 0 & 0 & 0 & 0 & 1\end{array}\right)}{\longrightarrow} k^{6} \stackrel{\left(\begin{array}{lllll}1 & 1 & 1 & 1 & 1\end{array}\right)}{\longrightarrow} k \rightarrow 0$.

The chains $p_{1}$ and $p_{2}$ satisfy condition (7.2) in Theorem $7.1(2)$. Hence $\mathbf{V}$ is a common frame for $\mathbf{F}$ and $\mathbf{F}^{\prime}$. Thus, the $M^{\prime}$-homogenization of $\mathbf{V}$ is the minimal free resolution of $V / M^{\prime}$.

\section{ACKNOWLEDGMENTS}

The first author was partially supported by NSF. We thank Jeff Mermin and Mike Stillman for helpful discussions. We are grateful to the TAMS referee for a very helpful report.

\section{REFERENCES}

[1] E. Batzies and V. Welker: Discrete Morse theory for cellular resolutions, J. Reine Angew. Math. 543 (2002), 147-168. MR.1887881 (2003b:13017)

[2] D. Bayer, I. Peeva, and B. Sturmfels: Monomial resolutions, Math. Research Letters 5 (1998), 31-46. MR1618363(99c:13029) 
[3] D. Bayer and B. Sturmfels: Cellular resolutions, J. Reine Angew. Math. 502 (1998), 123140. MR1647559 (99g:13018)

[4] T. Clark: Poset Resolutions and Lattice-Linear Monomial Ideals, J. Algebra 323 (2010), no. 4, 899-919. MR2578585

[5] H. Charalambous and A. Tchernev: Free resolutions for multigraded modules: A generalization of Taylor's construction, Math. Res. Lett. 10 (2003), 535-550. MR1995792 (2004e:13020)

[6] S. Eliahou and M. Kervaire: Minimal resolutions of some monomial ideals, J. Algebra 129 (1990), 1-25. MR1037391 (91b:13019)

[7] V. Gasharov, T. Hibi, and I. Peeva: Resolutions of a-stable ideals, J. Algebra 254 (2002), 375-394. MR1933875 (2003j:13014)

[8] V. Gasharov, I. Peeva, and V. Welker: The lcm-lattice in monomial resolutions, Math. Research Letters 6 (1999), 521-532. MR 1739211 (2001e:13018)

[9] M. Jöllenbeck and V. Welker: Resolution of the residue field via algebraic discrete Morse theory, Memoirs of the AMS, Volume 197, Number 923, 2009. MR2488864 (2009m:13017)

[10] J. Mermin: The Eliahou-Kervaire resolution is cellular, preprint.

[11] E. Miller: The Alexander duality functors and local duality with monomial support, $J$. Algebra 231 (2000), 180-234. MR1779598 (2001k:13028)

[12] G. Kalai: $f$-vectors of acyclic complexes, Discrete Math., 55 (1985), 97-99. MR0793634 (86k:52010)

[13] A. Tchernev: Representations of matroids and free resolutions for multigraded modules, Adv. Math. 208 (2007), no. 1, 75-134. MR2304312(2008i:13020)

[14] M. Velasco: Minimal free resolutions that are not supported by a CW-complex, J. Algebra 319 (2008), 102-114. MR2378063(2008j:13028)

Department of Mathematics, Cornell University, Ithaca, New York 14853

Department of Mathematics, Cornell University, Ithaca, New York 14853 